International Journal of General Systems, Vol 35 (3), pp. 377-386. 2006.

\title{
Analysis and control of complex collaborative design systems
}

\author{
S.F. Qin and Guangmin Sun* \\ School of Engineering and Design, Brunel University, UK \\ *Department of Electronic Engineering, Beijing University of Technology, China
}

\begin{abstract}
s:
This paper presents a novel method for modelling the complexity of collaborative design systems based on its analysis and proposes a solution to reducing complexity and improving performance of such systems. The interaction and interfacing properties among many components of a complex design system are analysed from different viewpoints and then a complexity model for collaborative design is established accordingly. In order to simplify complexity and improve performance of collaborative design, a general solution of decomposing a whole system into sub-systems and using unified interface mechanism between them has been proposed. This proposed solution has been tested with a case study. It has been shown that the proposed solution is meaningful and practical.
\end{abstract}

Keywords: Complex system, collaborative design, complexity model, complexity reduction, improvement of system performance.

\section{Introduction}

Today, economic globalisation is creating competitive pressures on industry to minimise the time to bring products to market. Information technologies and the World Wide Web are changing the way business enterprises work. Competing suppliers, designers, manufacturers and customers form a link via Internet or traditional media in 
new product development (NPD) where the dynamics and uncertainty rule out any centralised control. Collaborative design plays a key role in new product development (Jassawalla and Sashittal 1995). Collaboration is described as a more complex, higher intensity cross-functional and inter-organizational linkage. Participants may be distributed geographically over the world and belong to different organisations with variety of cultures, skills and etc. In a complex collaborative design system, participants are intricate organizational, technological and financial meta-systems operating under dynamic market conditions and uncertain business circumstances. Collaborative design system is characterized by very high complexity and is typically heterogeneous and very dynamic, involving complex interactions among many humans, applications, services, and devices.

Considerable research efforts have been devoted to understanding the importance of the collaborative design process, its complexity, and the resulting managerial challenges to make NPD and collaborative design process more efficient and effective. In recent years, several new ways have been suggested for improving collaborative design process such as the use of cross-functional teams (Edward and McDonough 2000), computer supported collaborative design tools (Wang et al. 2002, Fuh and Nee 2004), user-centered design methodology (Veryzer and Mozota 2005), supplier involvement (Petersen et al. 2003), and interfirm modularity strategy (Standenmayer et al. 2005). However, relatively little research has been focused specifically on quantitatively measuring the complexity of a collaborative design system and its implication. This makes system comparisons and evaluations uneasy in a quantitatively way. 
This article first analyses the collaborative design systems in NPD context and then introduces a complexity model for them. The model can be further used for comparing different collaborative design systems and their management. In order to simplify complexity and improve performance of collaborative design, a general solution of decomposing a whole system into sub-systems and using unified interface mechanism between them has been proposed. This proposed solution has been tested with a case study. It has been shown that the proposed solution is meaningful and practical.

The rest of the paper is organised as follows. Analysis of complex collaborative design system is presented in Section 2 and followed by the proposed complexity modelling in Section 3. In Section 4, the strategy for reducing the complexity of collaborative design is suggested and its implementation is exemplified with a case study. Finally, conclusions are drawn in Section 5.

\section{Analysis of Complex Collaborative Design Systems}

Collaborative design is a complex system involving people, technologies and processes. It is performed by multiple participants—representing individuals, teams or even entire organizations — each potentially capable of proposing values for design issues and/or evaluating these choices from their own particular perspectives. The design activities are influenced not only by the technological factors, but also by the interactions among various participants with different perspectives. In order to identify the complexity of a collaborative design system, analysis on the constituent participants, the system structure, design processing, design behavioral and dynamic features has been conducted as follows: 


\subsection{Constituent participants}

Collaborative design participants may include artists, industrial designers, engineering designers, manufacturing engineers, business managers, suppliers, customers, and etc. For example, artists can work at the conceptual design stage to perform aesthetical design of product. The industrial designers can produce product form design, ergonomics evaluation, human factors, and address environmental issues and branding strategies. Engineering designers can detail product and make it realizable. Manufacturing engineers can credit its manufacturability. Recently, suppliers and customers are brought into the design team. Petersen et al. (2003) has shown that material and component supplier involvement on collaborative design generally results in a higher achievement of NPD team goals. This involvement may range from simple consultation with suppliers on design ideas to making suppliers fully responsible for the design of materials, components or systems they will supply. Furthermore, the benefits from the users (or customers) involvement in product design and development have been identified (Veryzer 2005) in terms of enhancing collaborative new product development, improving idea generation, producing superior product or service solutions, and facilitating product appropriateness and adoption. Of course, any design project needs to be managed properly. Business and project managers at different levels such as institutional, departmental and team managers are key participants as well. All those participants may have differences in educational and cultural background, professional knowledge and skills, personalities, and etc. The large number of participants and their differences above raise the complexity in interaction and interfacing.

\subsection{Technical complexity}


A collaborative design process may result in a very complex product (or a system) such as a rocket or a car. It may also terminate in engineering artifacts such as the Three Georges Dam in China. The resultant artifacts may involve various techniques. For example, a rocket may have 40,000 electronic components and devices, and 4000 parts related to 150 equipments. Technically, designing a complex artifact needs various sciences, e.g., economics, sociology and management, and engineering technologies such as nuclear, control, mechanical and manufacturing engineering. Various tools and techniques need to be integrated in a collaborative design process. People working at different scientific fields may use the same technical terms but mean different things, or vice versa. The technical complexity also presents a great challenge for design management of a multidisciplinary team.

\subsection{Social Interaction}

In collaborative design, the design process consists of not only technical decisionmaking but also social interaction because the participants in a design team play both technical roles and social roles (Lu and Cai 2001). Technical activities are concerned with "what" and "how" issues such as developing the function structure for the product, searching or generating the product infrastructure options, and assigning the values to the design parameters, while the social interaction deals with "why" and "who" issues. It provides the negotiations among collaborative design decisions. The technical roles are depended on specialized expert knowledge and skills, while the social roles are normally influenced by the organization structure, norm, and culture. Based on their roles, the ways the participants understand design and manipulate their activities are not uniform. They usually adjust the attitudes based on the feedback from others. During 
social interactions, the participants usually solve their conflicts and negotiate design constraints, objectives and outcomes. Meanwhile, these interactions will change participants' perspectives and affect their technical decisions. Thus, the collaborative design process becomes more complicated than individual design.

\subsection{Creativity}

Design is one of the most human-related activities featuring intellectualism, creativity and ingenuity. Especially, at the crucial conceptual stage, design process is a relatively unstructured, consisting of high cognitive and non-routine activities with spare methodological support. Conceptual design has the characteristics of fuzzy problems, tolerating a high degree of uncertainty. Conceptual design issues at stake are highly interdisciplinary, and often involve collaboration from customers, designers and engineers. Designers often generate ideas and turn them into quick sketches with basically $2 \mathrm{D}$ tools like pencil and paper, while at the same time these activities are guided by function design and certain business processing. When they view their design sketches and play with them, new ideas come into their mind. It seems that the creativity depends on the individual efforts. Actually, making new creations depends on the ability of participants to collaborate with others, to understand and accept differences. Collaboration involves synergy (emergent property of complex system)that is, the design outcomes exceed the sum of the capabilities of the individual participants in the design process. They emerge from the interaction of constituent

participants. A good collaboration depends on participants who contribute an openness to change, willingness to cooperation and a high level of trust. 
The organisation factor affects the level of collaboration such as the priority the senior management gives to collaborative design and the level of autonomy afforded to participants in the design process. A collaborative design task normally is decomposed into sub-tasks. For each subtask, it may be sub-contracted to a design institution (a company, or a group of people or an individual). This task decomposition structure (or job distribution structure) has a hierarchy from the host institution down gradually to its guest institutions (sub-contractors). Each institution is an intricate organizational, technological and financial meta- collaboration design system and has its own unique viewpoints and circumstances such as financial situations and laws. For the same object, they may have different perceptions and make different decisions. Due to the involvement of human beings, the design process is not only based on the natural law of the artifact but is also affected by institutional and people's goals, skills, and circumstances. In fact, it is impossible to completely share knowledge and purpose among institutions (or designers) in collaborative design. For example, each institution wants to protect their intellectual properties (IP protection); therefore, some design information cannot be shared. Thus, interfacing between them is a complex issue.

\subsection{Design knowledge acquisition}

Marsh and Stock (2003) have shown that the re-use of previous design experience, knowledge, and models can be beneficial to a new design project. However, gathering and transforming information and knowledge from prior design projects so that it can be used in future development projects requires good management commitments. For example, project audits, design databases, design models (CAD/CAM/CAE), engineering notebooks, collections of test and experimental results, market research, project management databases, and other activities will all be important in the 
acquisition of knowledge from prior design projects. By the nature of collaboration, the organizational institutions for a collaborative design are heterogeneous and very dynamic. Therefore, there is an interfacing problem in gathering design information and reusing it in a new project.

\subsection{Conflicts}

As mentioned before, a collaborative design is a technical decision making process coupled with social interactions. Therefore, within the collaborative design process, design information and decision-making are driven by social, technological, scientific, and interdisciplinary dependencies. During this process, there are a lot of conflicts to be well managed such as decision conflicts between different participants, information inconsistence due to the use of different design models and tools, and communication barriers due to language, culture and technical differences. Although there are some researches focused on developing computer-mediated communication (CMC) and computer-supported cooperative work $(\mathrm{CSCW})$ tools, the effective conflict management and negotiation tools are still needed. For example, how a computer-based tool can provide face-to-face conversation environment to improve participants' confidence and trust to the others.

\subsection{Performance evaluation}

In order to manage collaborative design, design performance should be measured against its complexity, efficiency, effectiveness, sustainability, social proudness, emotional happiness, environmental effects, and etc. The complexity measurement will make collaborative design comparable. Good individual participants working together don't always form a good collaborative design teams depending on their fitness to each 
other. Similarly, the assembling result from good individual artifacts may be not a good collaborative design product depending on how they can fit to each other. This complex system feature makes performance evaluation difficult.

\subsection{Research complexity}

Complex systems have drawn much attention (Mitleton-Kelly 2003, Bossomaier and Green 2000) from science and engineering research (Kemal 2005). They have multiple interacting components whose collective behaviour cannot be simply inferred from the behaviour of individual components. The recognition that understanding the parts cannot explain collective behaviour has led to various new concepts and methodologies that are affecting all fields of science and engineering, and are being applied to technology, business and even social policy. Surrounded by Nature, we have looked into Nature for useful and inspirational lessons, analogies and similar behaviours. Scientists looked into Nature to analyse and understand, while engineers looked into Nature to synthesize and create. Understanding the entire context, guessing behavioural dynamics under an endless flow of events and pressure of data streams poses several grand challenges. They are still awaiting the major breakthroughs in the science of complex systems enabling thus advances of engineering practice.

In Engineering, International Federation of Automatic Control (IFAC) has a Technical Committee on Large Scale Complex System (TC 5.4). In this research community, large-scale systems have been traditionally characterized by a large number of variables, nonlinearities and uncertainties. Their decomposition into smaller, more manageable subsystems, possibly organized in a hierarchical form, has been associated with intense and time-critical information exchange and with the need for efficient co-ordination 
mechanisms. The TC now focuses on manufacturing and related systems characterized by a large number of variables, nonlinearities, uncertainties, and/or a networked structure of interconnected subsystems. It aims at developing new hierarchical control methods, decision-making and risk analysis techniques together with practical solutions based on new advances in computer and communication tools.

In collaborative design, researches focus on individual elements such as design communication, design modelling and design management. But there is only a little research focusing on collaborative design with a complex systems perspective (Wang et al 2002, Klein et al 2002).

\section{Complexity in Collaborative Design}

Collaborative design is a process to result in physical artefacts such as cars, rockets, washing machines and chairs, as well as informational artefacts such as software, organizations, business processes, services and plans and schedules. The design is performed by many participants (representing individuals, teams or even entire organisations) with different tools, knowledge, languages, methodologies and processes working on different elements of the design. All participants interact with others interdependently. How do we measure the complexity in collaborative design?

Complexity is defined as the amount of information necessary to describe the system (Klein 2002, Bar-Yam 2005). Here the complexity of a system is divided into five categories: the system complexity, algorithm complexity, behaviour complexity, designmaking complexity and negotiation complexity. For system complexity, obviously, it depends on the scale at which the system is described, however, once a particular scale is chosen the complexity should be well defined and bounded (at a particular instance) 
by the information necessary to describe the microstate of the system (the entropy), i.e. the number of design individuals involved, budgets, previous experience, the number of layers in decision-making and available design tools. Algorithm complexity relates to design technical issues such as issues in modelling, computation and communication. Behaviour complexity can be regarded as the amount of actions in response to its environment. Decision-making complexity depends on the number of decisions to be made to complete tasks and the amount of information needed to support decisionmaking. Negotiation complexity defines the amounts of negotiations having to be made with collaborators.

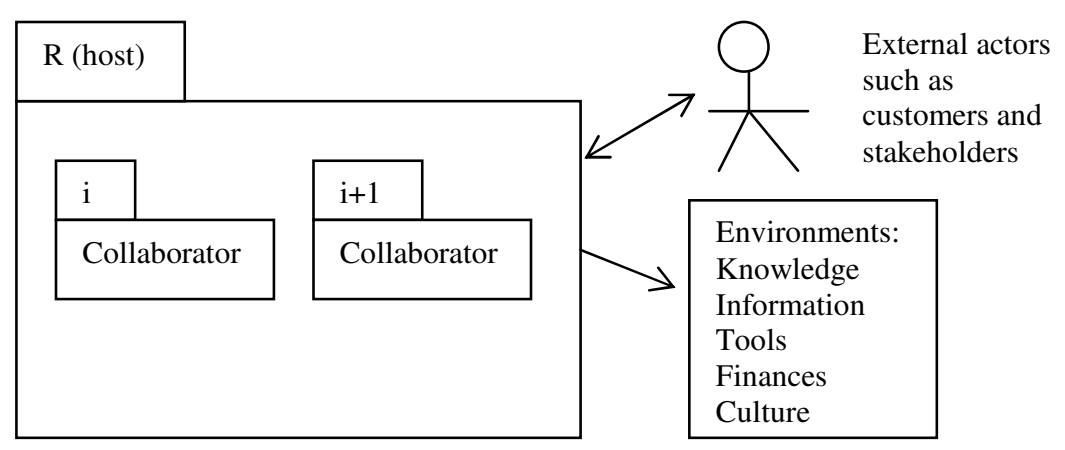

Figure 1. A UML model of Collaborative Design

Collaborative design is performed within a hierarchical business process. It can be represented as a network. Normally, a host organisation sets up a collaborative design task and produces design specifications based on initial marketing and business environments. The host organisation can be regarded as a UML package ' $R$ ' on the UML collaboration model (Figure 1). It has interactions with external actors such as customers and stakeholders, and environmental constraints such as financial and cultural issues. The whole design task can be then divided into sub-design tasks by the host organisation. These sub-tasks are contracted to different collaborators. Each 
collaborator is represented as another package node ' $i$ ' in Figure 1. Similarly, each collaborator can have their sub-contracts to other collaborators. Each collaborator as a node in the model can represent an individual, a team or even an entire organisation. The link between two nodes represents negotiations between them. The negotiation can have two states: 0 (no interactions between them or interactions completed), 1 (preparing for next negotiation or in current negotiation). Based on this collaborative design model, the node $i$ at time $t$ has a complexity as

$$
C_{i}(t)=C S_{i}(t)+C A_{i}(t)+C B_{i}(t)+C D_{i}(t)+C N_{i}(t)
$$

where $\mathrm{CS}, \mathrm{CA}, \mathrm{CB}, \mathrm{CD}$, and $\mathrm{CN}$ represent the system, algorithm, behaviour, decisionmarking and negotiation complexities respectively. For an overall collaborative design system with $n$ collaborators, its complexity at time $t$ is the sum of the components' complexity in connection with negotiation states. That is,

$$
C(t)=\sum_{i=1}^{n} \sum_{j=1}^{k} C_{i}(t)^{*} S_{i, j}(t)
$$

where, $\mathrm{k}$ is the number of links of the node $i$ at time $t . S_{i, j}(t)$ is the node negotiation state of its $j$ link.

This complexity model can represent dynamics of a collaborative design system. It can reflect the growth and shrinking phenomena of a nature system. It will start with 0 complexity, then increase nonlinearly, and finally shrink to 0 again when design is completed, all negotiation states will become 0 . Its dynamics is illustrated in Figure 2. 


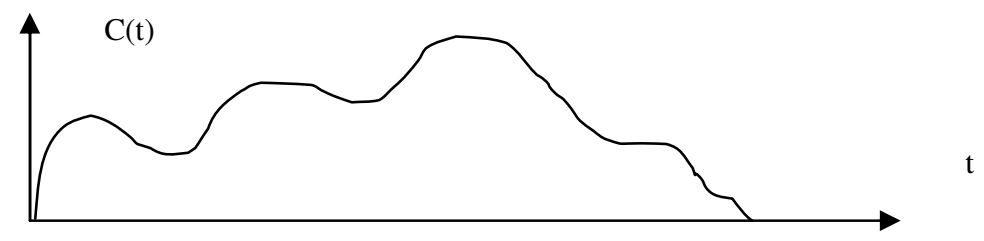

Figure 2: Illustration of complexity dynamics

\section{Reduction of complexity by simplifying interaction interfaces}

In order to reduce the complexity of collaborative design system, one strategy is to simplify interaction interfaces between participants and make communication and negotiation between them more efficient and effective. This can be achieved by utilizing unified interaction interface, which will provide a common communication and negotiation platform between participants. It will be associated with all key information for decision-making. Conceptually, this strategy can be supported by using intelligent agent technologies (Klein 2003). Here, an example of using unified interface into a mechanical system design is given below for demonstrating this strategy.

In this example, a unified interface is defined as a special assembly feature that unifies various assembly constraints into a form including a positional vector, a normal vector, a reference plane and a rotation angle about the normal vector. It also specifies interface and status information symbolically such as linking to a parent component or a child component, or a neutral component. Based on this interface mechanism, a product consisting of many components can be represented in a hierarchical structure (Fig. 3). 
The root node represents a product. The child node represents a component or a subassembly.

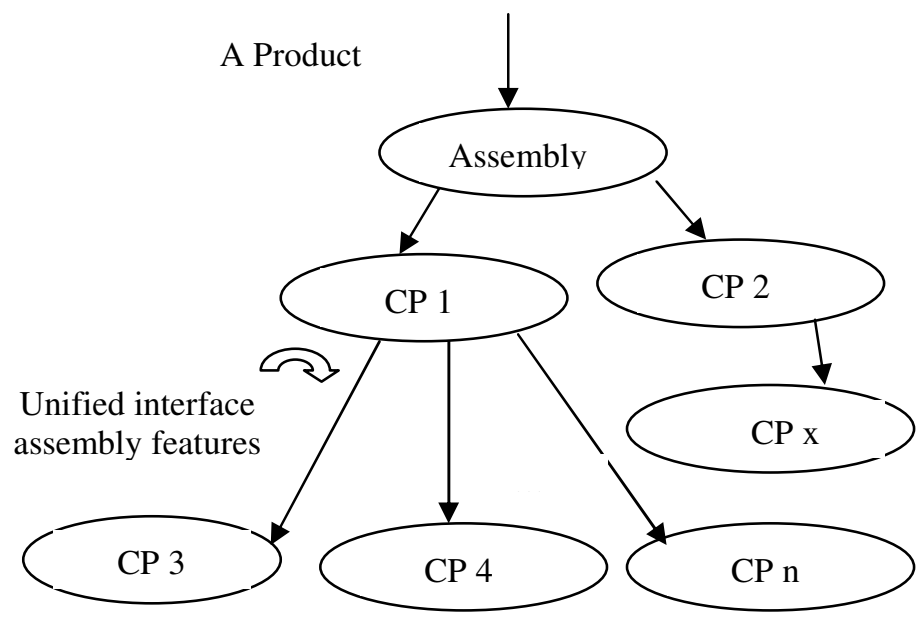

Figure 3. A product representation

This representation scheme focuses on a high-level "connect to" relation. Each component has well-defined functions, hierarchy and geometry. It can be highly selforganised. For example, they can be simulated over the Internet. Thus, assembly relationships exist only between a parent component and its child components. When creating an assembly, the user only needs to drag-and-drop a child node onto its parent node in the modelling tree. The user doesn't need to operate on detailed geometric information such as points, edges and faces as in typical CAD systems to constrain the assembly. By simplifying the unified assembly interface, assembling one parent component and one of its child components can be done automatically because the 
information defined in the unified interfaces can be used for calculating a relative position of the child component with respect to its parent.

After an assembly operation, status indicators in the related interfaces will be automatically checked to see if the unified interface is already occupied (assembled) or not yet. An initial status value for an interface is 0 , which means there are no assemblies linked to it. It is available to conduct an assembly. After assembling, the status value will be automatically changed to 1 , indicating the interface is occupied and is not available for any assemblies. This status indicator will provide an intelligent solution for solving over-assembly (conflict) problems by guaranteeing one interface with only one associated assembly.

A case study illustrates an assembly of its components. The assembly consists of the following components: Wing-Base, Transfer-Bar, Fixture, Top-Clamp, Feed-Slide, Cross-Slide, and Drill-Head. The assembly hierarchy is shown in Fig. 4. The links between two components are unified interfaces. After specifying the assembly model (Fig.4), the corresponding assembly model can be automatically generated and outputted as a VRML model (Fig 5).

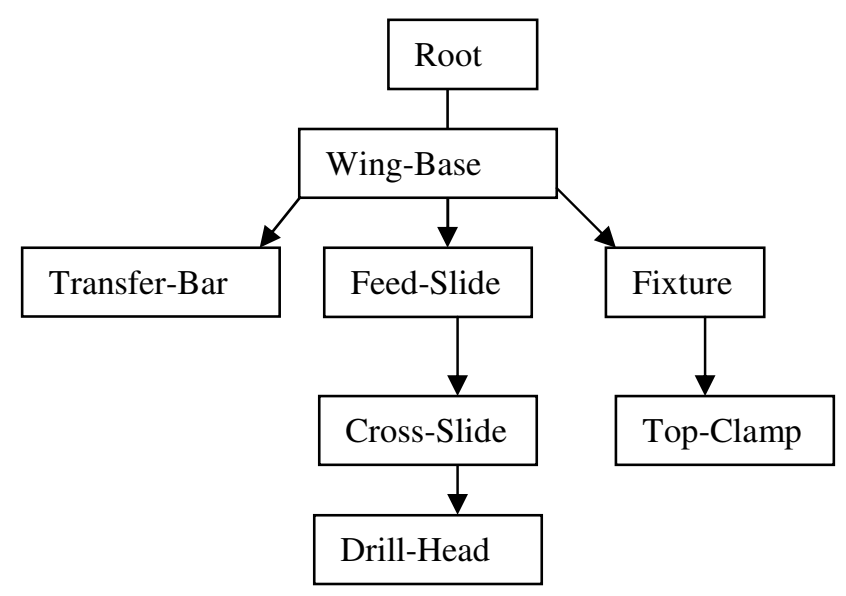

Figure 4. An example of assembly hierarchy 


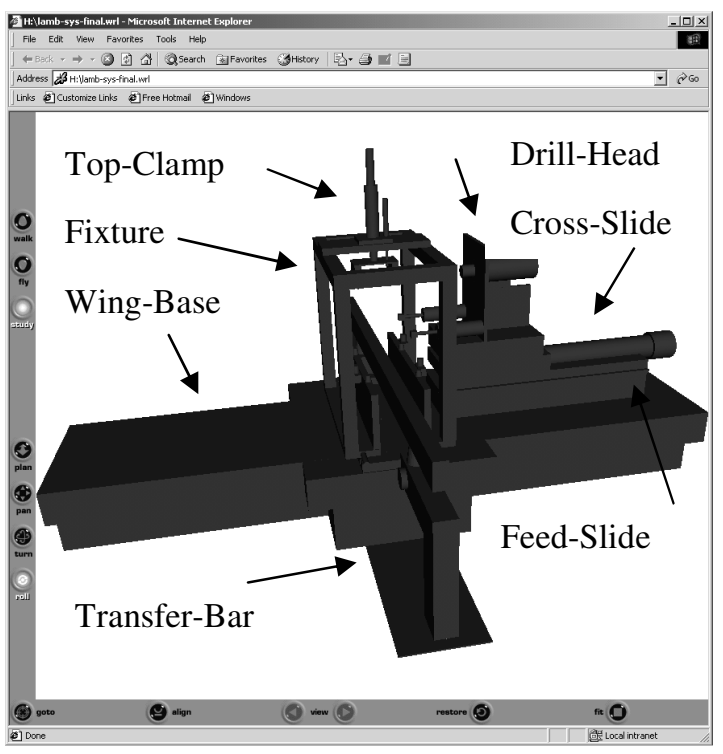

Figure 5. Drag-and-drop assembly

\section{Conclusion}

This paper regarded collaborative design as a complex system and analysed the complexity of collaborative design. In order to make collaborative design comparable, a new numerical model for measuring the complexity in collaborative design has been proposed. Based on this complexity model, a strategy of simplifying interaction interface was recommended to reduce the communication and interaction complexity of collaborative design. This strategy could be realised with current agent technologies (Klein 2003). An implementation of the strategy into a mechanical design system has been demonstrated through a case study, in which the concept of the unified assembly interaction interface was utilised to make assembly design simpler and easier.

\section{Reference}


K.J. Petersen, R.B. Handfield, and G.L. Ragatz, A model of supplier integration into new product development, J. Product Innovation and Management, 20, pp. 284-299, 2003.

A.R. Jassawalla and H.C. Sashittal, An examination of collaboration in HighTechnology new product development processes, J. Product Innovation and Management, 15, pp. 237-254, 1995.

S.J. Marsh and G. N. Stock, Building Dynamic Capabilities in New Product Development through Intertemporal Integration, J. Product Innovation and Management, 20, pp. 136-148, 2003.

F. Edward and III McDonough, Investigation of Factors Contributing to the Success of Cross-Functional Teams, J. Product Innovation and Management, 17, pp. 221$235,2000$.

J.Y.H. Fuh, A.Y.C. Nee, Distributed CAD for supporting Internet collaborative design. J. Computer-Aided Design, 36, pp. 759-760, 2004.

N. Staudenmayer, M. Tripsas, C. L. Tucci, Interfirm Modularity and Its Implications for Product Development, J. Product Innovation and Management, 22, pp. 303-321, 2005.

S.C.Y. Lu and J. Cai, A collaborative design process model in the sociotechnical engineering design framework, J. Artificial Intelligence for Engineering Design, Analysis and Manufacturing, 15, 3-20, 2001.

E. Mitleton-Kelly, Complex Systems and Evolutionary Perspectives on Organisations The Application of Complexity Theory to Organisations,Pergamon,London, 2003.

T. Bossomaier, D.G Green, Complex Systems, Cambridge University Press, 2000.

A.D. Kemal, Science and Engineering of Large-Scale Complex Systems, Ubiquity, Issue 7, March 15,2005.

L. Wang. W. Shen, H. Xie, Neelamkavil J. and Pardasani A., Collaborative conceptual design-state of the art and future trends, Computer-Aided Design, 2002: 34 981-996.

M Klein, S. Hiroki, F. Peyman and Y. Bar-Yam, A complex systems perspective on computer-supported collaborative design technology, Communications of the ACM, 2002, 45(1):27-31.

Y. Bar-Yam, http://necsi.org/projects/yaneer/points.html,2005

M. Klein, H. Sayama, P. Faratin, Y. Bar-Yam, dynamics of collaborative design: Insights from complex systems and negotiation research, Concurrent EngineeringResearch 11 (3): 201-209 SEP 2003 


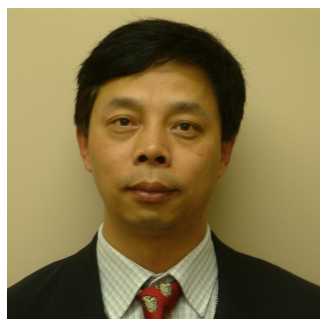

Shengfeng Qin is a Lecturer in the School of Engineering and Design, Brunel University, UK. He obtained his BSc and MSc degrees from China. He was an academic visiting scholar within the Geometric Modelling group at the University of Birmingham. After working as a Research Assistant at UWIC, he gained his PhD in Computer-Aided Design from the University of Wales. He subsequently joined the Manufacturing System Integration Research Institute (MSI), Loughborough University as a Research Associate. His main research interests are in computer aided conceptual design, sketch-based interface and modeling, and distributed collaborative design.

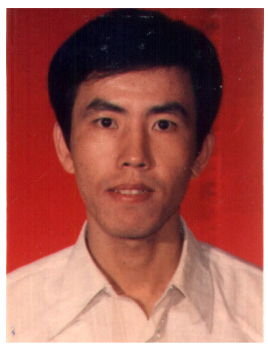

Guangmin SUN received a BSc in electronic engineering from Beijing Institute of Technology, China, in 1982, an MS in communication and information systems from Nanjing University of Science and Technoloty, China, in 1991, and a $\mathrm{PhD}$ in communication and information systems from Xidian University, China, in 1997. Dr SUN is a professor in the Department of Electronic Engineering at Beijing University of Technology. He is currently a visiting professor in the School of Engineering and Design at Brunel University, UK. His research interests include neural networks and applications, artificial intelligence, pattern recognition, bioinformatics, signal and image processing. 